\title{
Liquid chromatography - tandem mass spectroscopic method for the determination Of zerumbone in human plasma and its application to Pharmacokinetics.
}

\begin{abstract}
A rapid, sensitive, specific and selective LC-MS/MS method for the determination of zerumbone (ZER) in human plasma using 2,4-diamino-6-(4-methoxyphenyl)-1,3,5-triazine (DMTZ) as an internal standard (IS) has been developed and validated. ZER was chromatographed on $\mathrm{C} 8$ column using a mobile phase of acetonitrile/water $(80: 20, \mathrm{v} / \mathrm{v})$ at a flow rate of $0.25 \mathrm{ml} \min (-1)$. Quantitation was achieved using ESI+ interface, employing multiple reaction monitoring (MRM) mode at $\mathrm{m} / \mathrm{z} 219>81$ and $218>134$ for ZER and IS, respectively. The calibration standards were linear over a range of 5-3000 ng $\mathrm{ml}(-1)$ $(\mathrm{r}(2)=0.9994)$ with an LLOQ of $5 \mathrm{ng} \mathrm{ml}(-1)$ (RSD \%; 11.4\% and bias\%; 9.5\%). Intra- and inter-day precision of ZER assay ranged from 0.18 to $3.56 \%$ with accuracy (bias) that varied between -5.09 and $4.3 \%$, demonstrating good precision and accuracy. Recoveries of ZER and the IS from human plasma were above $85 \%$. The developed method was validated for the determination of ZER in rat plasma. Linearity, stability of ZER and the ME on rat plasma were discussed. The applicability of the developed method was demonstrated by measuring ZER in rat plasma samples following intravenous and intraperitoneal administration of ZER prepared in hydroxypropyl- $\beta$-cyclodextrin (HP $\beta C D$ ) and sodium carboxymethyl cellulose $(\mathrm{CMC})$, respectively, in $20 \mathrm{mg} \mathrm{kg}(-1)$ and this study indicated a clear significant difference $(p<0.05)$ in pharmacokinetic parameters of ZER in ZER/HP $\beta C D$ complex compared with ZER in CMC preparation.
\end{abstract}

Keyword: Tandem mass spectroscopic method; Zerumbone; Human plasma; Pharmacokinetics. 